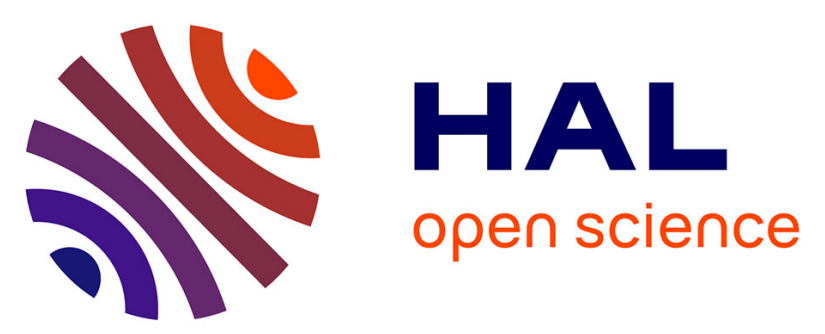

\title{
A Representation Theorem for Union-Difference Families and Application
}

\author{
Binh-Minh Bui-Xuan, Michel Habib
}

\section{To cite this version:}

Binh-Minh Bui-Xuan, Michel Habib. A Representation Theorem for Union-Difference Families and Application. LATIN: Latin American Symposium, Apr 2008, Búzios, Brazil. pp.492-503, 10.1007/978-3-540-78773-0_43. lirmm-00324969

\section{HAL Id: lirmm-00324969 \\ https://hal-lirmm.ccsd.cnrs.fr/lirmm-00324969}

Submitted on 16 Sep 2019

HAL is a multi-disciplinary open access archive for the deposit and dissemination of scientific research documents, whether they are published or not. The documents may come from teaching and research institutions in France or abroad, or from public or private research centers.
L'archive ouverte pluridisciplinaire HAL, est destinée au dépôt et à la diffusion de documents scientifiques de niveau recherche, publiés ou non, émanant des établissements d'enseignement et de recherche français ou étrangers, des laboratoires publics ou privés. 


\title{
A Representation Theorem for union-difference Families and Application ${ }^{\star}$ (Extended Abstract) ${ }^{\star \star}$
}

\author{
B.-M. Bui-Xuan ${ }^{1}$ and M. Habib ${ }^{2}$ \\ 1 CNRS - LIRMM - Université Montpellier II \\ 161 rue Ada, 34392 Montpellier Cedex 05, France. buixuan@lirmm.fr \\ 2 CNRS - LIAFA - Université Paris Diderot \\ Paris 7 Case 7014, 75205 Paris Cedex 13, France. habib@liafa.jussieu.fr
}

\begin{abstract}
We give a quadratic $O\left(|X|^{2}\right)$ space representation based on a canonical tree for any subset family $\mathcal{F} \subseteq 2^{X}$ closed under the union and the difference of its overlapping members. The cardinality of $\mathcal{F}$ is potentially in $O\left(2^{|X|}\right)$, and the total cardinality of its members even higher. As far as we know this is the first representation result for such families. As an application of this framework we obtain a unique digraph decomposition that not only captures, but also is strictly more powerful than the well-studied modular decomposition. A polynomial time decomposition algorithm for this case is described.
\end{abstract}

\section{Introduction}

Many combinatorial decompositions lead to interesting subset families, such as crossing families for minimum cuts in network flows theory (see e.g. [21]), and partitive families for modular decomposition in graph theory $[4,10,19]$. Crossfree families as defined in [21] using the famous Edmonds-Giles's theorem [9] admit a tree structure and arise in many combinatorial decompositions such as the split decomposition $[6-8,18]$ and also in phylogeny [22].

For a given set family $\mathcal{F} \subseteq 2^{X}$, it is worth studying its distance from a tree structure, namely to examine if it can be represented via a tree. Such a representation must allow the enumeration of all members of the family in $O(|\mathcal{F}|)$ time. Let us define the complexity of a family as the size of its minimal tree. At first level one can find simple hierarchies (c.f. laminar in [21]) and cross-free families. Then, (weakly) partitive families which admit a unique tree decomposition with 3 types of nodes (prime, complete and linear) also have complexity $O(|X|)$. For crossing families only a representation tree in $O\left(|X|^{2}\right)$ space is known [13].

This paper deals with union-difference families - families closed under the union and the difference of its overlapping elements - which is a natural generalisation of partitive families. We show the existence of a canonical tree representation in $O\left(|X|^{2}\right)$ space. Furthermore, from this we obtain a new polynomial and

^ Research supported by the ANR project Graph Decompositions and Algorithms.

${ }^{\star \star}$ Full version available at http://hal-lirmm.ccsd.cnrs.fr/lirmm-00175766. 
unique decomposition of directed graphs, generalising modular decomposition. A polynomial time decomposition algorithm for this case is then depicted in the last section.

\section{Representation Theorem}

Let $X$ be a finite set. Two sets $A$ and $B$ overlap, denoted by $A \oplus B$, if none among $A \cap B, A \backslash B$, and $B \backslash A$ is empty. They cross, if we have both $A \oplus B$ and $\bar{A} \oplus \bar{B}$, where $\bar{A}=X \backslash A$. A family $\mathcal{F} \subseteq 2^{X}$ is a union-difference family if: $\mathcal{F}$ contains the trivial members $X$ and $\{x\}$ (for all $x \in X$ ), and $\mathcal{F}$ is closed under the union and the difference of its overlapping members. If a union-difference family is also closed under the symmetric difference of its overlapping members, then it is closed under the intersection of its overlapping members too. Union-differenceintersection families are well-studied under the name of partitive families [4], and are fundamental for modular graph decomposition $[10,19]$.

Henceforth $\mathcal{F}$ is a union-difference family. Notice that if $|X| \leq 2$ then $\mathcal{F}=2^{X}$ and representing $\mathcal{F}$ is trivial. We assume throughout the paper that $|X| \geq 3$. $A \in \mathcal{F}$ is a strong member of $\mathcal{F}$ if $A$ does not overlap any $B \in \mathcal{F}$. Likewise, $A \in \mathcal{F}$ is a semi-strong member of $\mathcal{F}$ if it does not cross any $B \in \mathcal{F}$. Let $\mathcal{S} \subseteq \mathcal{F}$ be the family of semi-strong members of $\mathcal{F}$. For sake of simplicity, $X$ is excluded from $\mathcal{S}$ although it is clearly semi-strong. By definition, no two members of $\mathcal{S}$ cross, and $\mathcal{S}$ is called cross-free.

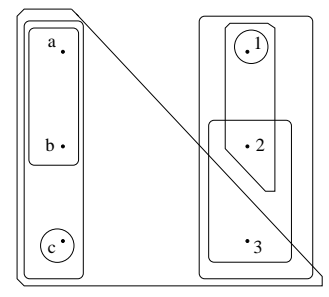

i.

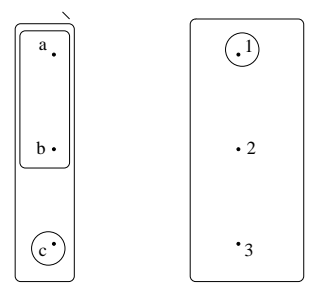

ii.
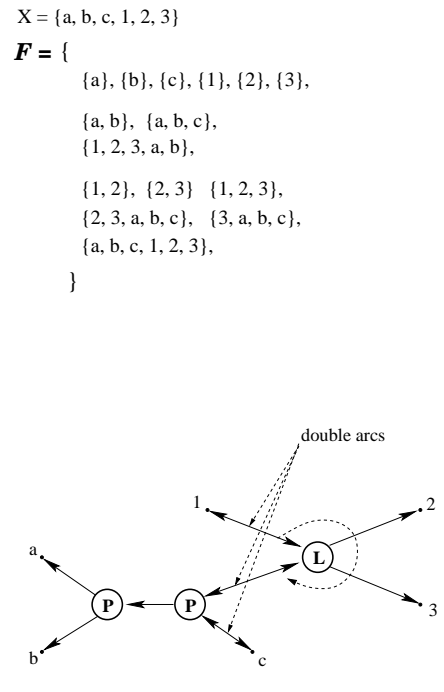

iii.

Fig. 1. i. A union-difference family, circles represent their complement. ii. The semistrong subfamily excluding $X$. iii. Decomposition tree. 
Let us now recall a cross-free family representation [9] which is widely used in combinatorial optimisation research areas (refer to e.g. [21]). Let $x \in X$, we consider $\mathcal{S}^{\prime}=\{A \mid A \in \mathcal{S} \wedge x \notin A\} \cup\{\bar{A} \mid A \in \mathcal{S} \wedge x \in A\}$. No two members of $\mathcal{S}^{\prime}$ overlap, and their inclusionwise ordering results in a tree rooted at $X \backslash\{x\}$. We then add $x$ to the children of the root and unroot the tree. The set of leaves is now in bijection with $X$ : by abusiveness we confound the two sets. In this tree, deleting any edge gives rise to two connected components. If each component is regarded as the set of its leaves, then at least one of them is a member of $\mathcal{S}$. Then, edge orientation can denote which ones belong to $\mathcal{S}$ (see Fig. 1). On the other hand, each member of $\mathcal{S}$ corresponds to one edge of the tree.

Definition 1 (Decomposition tree). We define the decomposition tree of a union-difference family $\mathcal{F} \subseteq 2^{X}$ as the Edmonds-Giles's tree representation [9] of its semi-strong members, $X$ is excluded. Such a tree has no degree 2 node.

We shall label this tree to obtain an enumerating object of all members of $\mathcal{F}$. In the tree, the deletion of an internal node $n$ gives rise to $k=d(n)$ connected components, which can also be seen as a $k$-partition of $X$. Let $\left\{X_{1}, \ldots, X_{k}\right\}$ denote this partition. For instance, the node labelled " $L$ " in Fig. 1 yields $\{\{1\},\{2\},\{3\},\{a, b, c\}\}$. For later use, notice that we always have $k \geq 3$. (This can also be seen as a quick proof that the unlabelled decomposition tree is of linear $O(|X|)$ size.) Let us consider $Y=\left\{X_{1}, \ldots, X_{k}\right\}$ as a set, and define the quotient of $\mathcal{F}$ with respect to node $n$ as the family $\mathcal{Q}(n) \subseteq 2^{Y}$ such that

$\left\{\left\{X_{i}\right\}\right.$ belongs to $\mathcal{Q}(n)$ for all $1 \leq i \leq k$,

$\left\{Q=\left\{X_{i} \mid i \in I\right\}\right.$ with $|Q| \neq 1$ belongs to $\mathcal{Q}(n) \Leftrightarrow \cup_{i \in I} X_{i}$ belongs to $\mathcal{F}$.

The membership of $X_{i}$ in $\mathcal{F}$ (resp. exclusion of $X_{i}$ from $\mathcal{F}$ ) is already stored by the edge orientation of the decomposition tree. Roughly, each member $Q$ of the quotient $\mathcal{Q}(n)$ corresponds to one and only one member of $\mathcal{F}$, except for the singletons $\left\{X_{i}\right\}$. Moreover, it is not obvious but folklore that the converse holds:

Proposition 1. For all member $A \in \mathcal{F}$ of a subset family $\mathcal{F} \subseteq 2^{X}$, there exists a node $n$ in the semi-strong tree of $\mathcal{F}$ such that $A$ corresponds to a member of the quotient $\mathcal{Q}(n)$ of $\mathcal{F}$ with respect to $n$. This node is unique.

Consequently, if there is a way to describe $\mathcal{Q}(n)$ for every node $n$ of the decomposition tree, then one can rebuild the initial family $\mathcal{F}$ in an exact manner. As a step towards this aim, we say that a member $A$ of $\mathcal{F}$ is quasi-trivial if $|A|=|X|-1$, and notice a second non obvious but folklore fact:

Proposition 2. Trivial and quasi-trivial members are semi-strong by vacuity. On the other hand, any semi-strong member of a quotient $\mathcal{Q}(n) \subseteq 2^{Y}$ is either trivial or quasi-trivial.

Remark 1. Both Propositions 1 and 2 hold for arbitrary subset families.

Definition 2 (Quotient property). We say that a subset family satisfies the quotient property if all its semi-strong members are either trivial or quasi-trivial. 
Obviously the quotient of a union-difference family is also a union-difference family. We thus focus on families satisfying both union-difference and quotient properties, which form a super-class of the quotient nodes of a union-difference decomposition tree. We shall prove that there are at most 5 types of them. Moreover, each type will be proved to be of "small enough" size, that is

Main Representation Theorem. There is a node-labelling of the decomposition tree of a union-difference family $\mathcal{F} \subseteq 2^{X}$ such that every member of $\mathcal{F}$ can be retrieved from the tree and its labels. Moreover, the size of the tree and its labels is in $O\left(|X|^{2}\right)$ space.

Proof. We shall consider two main categories. Simply-linked quotients (see further in Definition 3) will be characterised by Theorem 1 into 4 types. Section 2.2 addresses the remaining ones. Theorem 2 proves the quadratic global size.

Before continuing, let us highlight a useful tool from previous works on partitive families. A subset family $\mathcal{F} \subseteq 2^{X}$ can also be seen as an undirected hypergraph with vertex set $X$. Let us define the $2-$ graph of $\mathcal{F}$ as its restriction to size 2 hyperedges: $G_{\mathcal{F}}=(X, E)$ with $E=\{A \in \mathcal{F}$ and $|A|=2\}$. Though the following property was discovered for partitive families, its proof only requires the union and difference closures. (The proof given in [10] is recalled in the full version [1].)

Lemma 1. (c.f. $[4,10]$ with partitive families) Let $\mathcal{F}$ be a union-difference family. If its $2-$ graph $G_{\mathcal{F}}$ is connected then $G_{\mathcal{F}}$ is either a clique, a path, or a cycle.

\subsection{Simply-linked quotients}

We first focus on a case of "easy" decomposition, fully exploiting Lemma 1. While a quasi-trivial member is clearly semi-strong, it is not necessarily strong. Moreover, we say that

Definition 3 (Simply-linked Property). A subset family is simply-linked if none of its quasi-trivial members is strong.

For simply-linked quotients, the following nice theorem holds. A family is prime if it has only trivial and quasi-trivial members.

Theorem 1. If a union-difference family $\mathcal{F}$ satisfies both quotient and simplylinked properties, then one and only one of the following holds:

- $G_{\mathcal{F}}$ is a clique (we say that $\mathcal{F}$ is complete),

- $G_{\mathcal{F}}$ is a path (we say that $\mathcal{F}$ is linear),

- $G_{\mathcal{F}}$ is a cycle of length at least 4 (we say that $\mathcal{F}$ is circular),

- $\mathcal{F}$ is prime.

Proof. First we have to prove the two lemmas 2 and 3 (below). Then, notice by Lemma 1 that if $G_{\mathcal{F}}$ is connected, it is either a clique, a path, or a cycle. 
By union closure, $G_{\mathcal{F}}$ is a clique if and only if $\mathcal{F}=2^{X}$, and we say that $\mathcal{F}$ is complete. Likewise, $G_{\mathcal{F}}$ is a path (resp. cycle) if and only if there is a linear (resp. circular) ordering of $X$ such that $\mathcal{F}$ is exactly the family of all intervals (resp. circular intervals) of this ordering. $\mathcal{F}$ is then linear (resp. circular).

Corollary (Representing simply-linked quotients). Let $X_{1}, \ldots, X_{k}$ denote the resulting connected components of a decomposition tree when deleting a quotient node. Representing a complete quotient node is easily done with $O(1)$ label, stating the quotient is the family of every union of some $X_{i}^{\prime}$ 's. For a linear or circular node, we also need to code an ordering on the incident edges. Then, an $O(1)$ label can state the quotient is the family of every union of some consecutive $X_{i}$ 's (Fig. 1 gives an illustration on the node labelled " $L$ "). Except for the special case of $X$, members of a prime quotient node are already stored in the edge orientation of the decomposition tree (they are semi-strong, and belong to $\mathcal{S}$ ). Accordingly, we only need an $O(1)$ label for all prime nodes, stating there are no members bound to the node other than those given by the edge orientation.

Let us head back to the proof of Theorem 1. A chain of length $k$ of $\mathcal{F}$ is a sequence $\left(A_{1}, \ldots, A_{k}\right)$ of members of $\mathcal{F}$ such that $A_{i} \oplus A_{i+1}$ for all $i$, and $A_{i} \cap A_{j}=\emptyset$ for all $|i-j|>1$. The chain is covering if $A_{1} \cup \cdots \cup A_{k}=X$, and irreducible if $\left|A_{i}\right|=2$ for all $1 \leq i \leq k$. An irreducible and covering chain of $\mathcal{F}$ can also be seen as a Hamiltonian path in the 2 -graph $G_{\mathcal{F}}$, which would imply its connectivity, and enable the use of Lemma 1.

Lemma 2. If a union-difference family $\mathcal{F}$ satisfies both quotient and simplylinked properties, then either $\mathcal{F}$ is prime, or $\mathcal{F}$ has a length 3 covering chain.

Proof. Suppose that $\mathcal{F}$ is not prime, and let $A \in \mathcal{F}$ be neither trivial nor quasitrivial. We take $A$ maximal by inclusion. The quotient property provides us with $B \in \mathcal{F}$ such that $A$ and $B$ cross. The closure under union implies $A \cup B \in \mathcal{F}$. Moreover, $A$ is maximal. Hence $A \cup B$ is either trivial or quasi-trivial. However, $A \cup B$ cannot be trivial since $A$ and $B$ cross. Then, the simply-linked property implies that $A \cup B$ is not strong. Hence it overlaps some member $C \in \mathcal{F}$. Here, all cases lead to either $D=C \cup B \backslash A$ or $E=C \cup A \backslash B$ is a member of $\mathcal{F}$. Then, either $(A, B, D)$ or $(B, A, E)$ is a covering chain of length 3 .

Lemma 3. If a union-difference family $\mathcal{F}$ satisfies both quotient and simplylinked properties, and has a covering chain of length at least 3 , then $\mathcal{F}$ has an irreducible covering chain (then $G_{\mathcal{F}}$ is connected).

By lack of space, the proof of Lemma 3 is omitted. Please refer to the full version [1] for any detail.

\subsection{Other quotients}

We now address a family $\mathcal{F} \subseteq 2^{X}$ that is not simply-linked. By definition it has a quasi-trivial member that is strong. We note $Y=X \backslash\{x\}$ that member. Since 
$Y$ is strong, except for $X$ and $\{x\}, \mathcal{F}$ has no other member containing $x$. Let us consider the sub-family $\mathcal{G}=\mathcal{F} \backslash\{X,\{x\}\}$, which holds $\mathcal{G} \subseteq 2^{Y}$. Obviously, if $\mathcal{F}$ is a union-difference family, so is $\mathcal{G}$. Fainthearted, we represent $\mathcal{F}$ with the member $\{x\}$ and the union-difference decomposition tree of $\mathcal{G}$. We process the same way with all quotient nodes that are not simply-linked. Therefore, such a tree may have recursive levels. Fortunately enough, its total size still is polynomial:

Theorem 2. The global size of the labelled decomposition tree of a given uniondifference family $\mathcal{F} \subseteq 2^{X}$ is in $O\left(|X|^{2}\right)$.

Proof. By induction on $n=|X|$. Let $f(n)$ be the maximum size of all decomposition trees of $n$ leaves. Obviously, $f(1)$ and $f(2)$ are non null constants. Let $f(k) \leq \alpha \times k^{2}$ hold for all $k<n$. We suppose without loss of generality that $\alpha$ is greater than any other constant in this proof. Let us consider a decomposition tree of $n$ leaves and let $N$ be the set of its internal nodes. For each $i \in N$, let $n_{i}$ be its degree. The label of $i$ is either of constant size (c.f. prime and complete nodes), of linear size on $n_{i}$ (c.f. linear and circular nodes), or of size bounded by $f\left(n_{i}-1\right)+\beta$ (c.f. nodes that are not simply-linked). In all cases, it is bounded by $\alpha \times\left(n_{i}-1\right)^{2}+\alpha$ since $n_{i} \geq 3$ and $\alpha \geq \beta$. The total size of leaves, edges, and orientations is linear on $n$, hence bounded by $\alpha \times n$. We deduce that

$$
f(n) \leq \alpha \times\left(\sum_{i \in N}\left(\left(n_{i}-1\right)^{2}+1\right)+n\right) \leq \alpha \times\left(\sum_{i \in N}\left(n_{i}-1\right)^{2}+n^{\prime}+n\right),
$$

where $n^{\prime}=|N|$. Notice that $\sum_{i \in N} n_{i}=n+2 \times\left(n^{\prime}-1\right)$ (the $n$ pendant edges are counted once while other edges are counted twice). In other words, $S=$ $\sum_{i \in N}\left(n_{i}-1\right)=n+n^{\prime}-2$. Then, the greatest value that $\sum_{i \in N}\left(n_{i}-1\right)^{2}$ can reach happens when one among the $n_{i}$ gets the greatest value possible. Since $n_{i}-1 \geq 2$, we have $\sum_{i \in N}\left(n_{i}-1\right)^{2} \leq\left(n^{\prime}-1\right) \times 2^{2}+\left(S-\left(n^{\prime}-1\right) \times 2\right)^{2}$. Then, $f(n) \leq \alpha \times\left(n^{2}+n^{\prime 2}+5 n^{\prime}+n\left(1-2 n^{\prime}\right)-4\right)$. Besides, that there are no degree 2 nodes in the tree provides us with $n \geq n^{\prime}+2$. Finally, combining the previous facts and $1-2 n^{\prime} \leq 0$ allows to conclude.

Conjecture: There is for every union-difference family a representation of smaller space than quadratic on the size of the ground set. The reason for the conjecture comes from the brute-force aspects of this section.

\section{Application to Graphs: Sesquimodular Decomposition}

In graph theory modular decomposition is now a well-studied notion $[4,10,15$, $19]$, as well as some of its generalisations $[6-8,17,18,20]$. As having been rediscovered in other fields, the notion also appears under various names, including intervals, externally related sets, autonomous sets, partitive sets, and clans. Direct applications of modular decomposition include tractable constraint satisfaction problems [5], computational biology [14], graph clustering for network analysis, and graph drawing. This rich research field lays heavily on the nice 


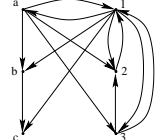

(a) The family of sesquimodules of this digraph is the one of Fig. 1 (refer therein for the decomposition tree). Notice that $\{2,3\}$ is also a module.

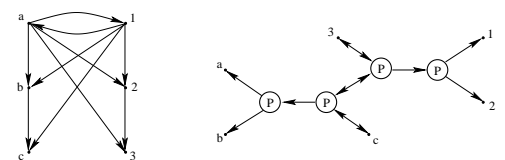

(b) A modular prime digraph with its sesquimodular decomposition tree.

Fig. 2. Modules v.s. sesquimodules.

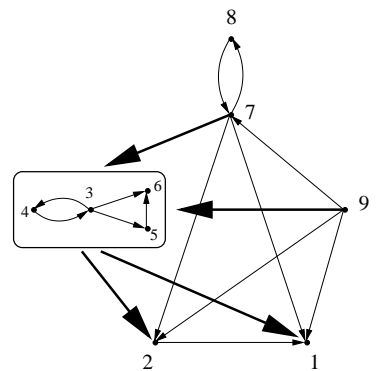

(a) A directed graph, bold arcs denote all-to-all arcs.

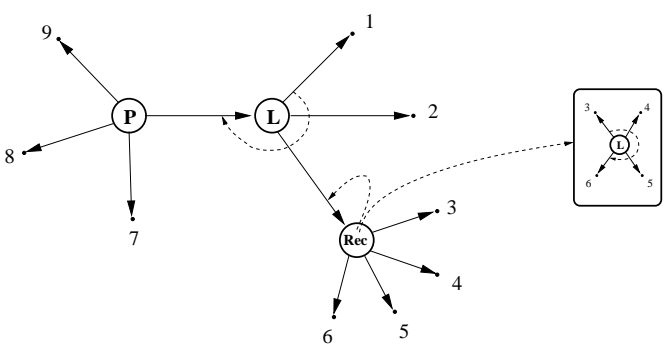

(b) Its sesquimodular decomposition tree.

Fig. 3. Sesquimodular decomposition. The family of non-trivial sesquimodules of the digraph is $\{\{1,2\},\{1,2,3,4,5,6\},\{2,3,4,5,6\},\{2,3,4,5,6,7,8,9\},\{3,4,5,6,7,8,9\}$, $\{3,4\},\{3,4,5\},\{3,4,5,6\},\{4,5\},\{4,5,6\},\{5,6\}\}$.

combinatorial properties of modules. Among most important ones, that modules form a partitive family allows representing them compactly with a tree [4, $10,19]$.

Besides, in the area of social networks, several vertex partitioning have been introduced in order to catch the idea of putting in the same part all vertices acknowledging similar behaviour, in other words finding regularities [23]. Modular decomposition provides such a partitioning, yet seemingly too restrictive for real life applications. The concept of a role [11] on the other hand seems promising, however its computation is unfortunately $N P$-hard [12]. As a natural consequence, there is need for the search of relaxed, but tractable, variations of the modular decomposition scheme. We here investigate the case of directed graphs, and propose a weakened definition of module in order to further decompose. Fortunately enough, we still obtain a well-structured variation, thanks to union-difference families.

Digraphs here refer to loopless simple directed graphs where 2-cycles are allowed. Let $G=(V, A)$ be a digraph, $M \subseteq V$ is a sesquimodule if:

$-\forall x, y \in M, N^{-}(x) \backslash M=N^{-}(y) \backslash M$, and

- $\forall x, y \in M$, either $N^{+}(x) \backslash M=N^{+}(y) \backslash M$ or $N^{+}(x) \backslash M=\overline{N^{+}(y)} \backslash M$.

In an undirected graph, there is only one requirement to be a module, which is $\forall x, y \in M, N(x) \backslash M=N(y) \backslash M$. The classical generalisation to directed graphs 
requires two full conditions, one on in-neighbours and one on out-neighbours: $\forall x, y \in M, N^{-}(x) \backslash M=N^{-}(y) \backslash M$ and $N^{+}(x) \backslash M=N^{+}(y) \backslash M$. In the new definition, there is a full condition on in-neighbours, and a relaxed one on outneighbours: the exterior still has to be partitioned into out-/non-out-neighbour vertices, however, their order is irrelevant. This is the reason for the terminology. Fig. 2(a) exemplifies an instance where the sesquimodules form the family given in Fig. 1, while Fig. 2(b) shows that the generalisation of modules to sesquimodules is proper. A more complex example of sesquimodular decomposition tree is given in Fig. 3. We have the following theorem.

Theorem 3 (Uniqueness Decomposition Theorem). There is a unique unrooted tree associated to a digraph $G=(V, A)$ such that: the leaves of the tree are in one-to-one correspondence with the vertices of $G$; the edges of the tree are oriented; the internal nodes of the tree are marked with at most 4 types of labels; and all sesquimodules of $G$ can be generated from this tree without the knowledge of the graph. The size of this tree and its labels is in $O\left(|V|^{2}\right)$.

This theorem lays on the simple fact that

Proposition 3. The sesquimodules of a digraph form a union-difference family. Furthermore there are no circular nodes in its decomposition tree.

Proof. Let $G=(V, A)$ be a digraph. Clearly, the trivial vertex subsets are sesquimodules of $G$. Let $X$ and $Y$ be two overlapping sesquimodules of $G$. It follows straight from definition that $X \cup Y$ is a sesquimodule. We only need to prove that $Z=X \backslash Y$ is also a sesquimodule.

First suppose that there exist an exterior vertex $s \notin Z$ and two vertices $x, y \in Z$ s.t. $(s, x) \in A$ and $(s, y) \notin A$. We shall denote arc $(x, y) \in A$ by $x y$, and non-arc $(x, y) \notin A$ by $\overline{x y}$. Since $X$ is a sesquimodule $s$ belongs to $X \cap Y$. Moreover, that $X$ and $Y$ overlap implies there is a vertex $t$ belonging to $Y \backslash X$. Notice that $s, t \in Y$ and $x, y \notin Y$. Additionally, we have $s x$ and $\overline{s y}$. Since $Y$ is a sesquimodule, we have either $t x \wedge \overline{t y}$ or $\overline{t x} \wedge t y$. But then $X$ no more is a sesquimodule as $t \notin X$ and $x, y \in X$. Hence, for all $x, y \in Z, N^{-}(x) \backslash Z=N^{-}(y) \backslash Z$.

Now let $x, y \in Z$ and $s, t \notin Z$. For convenience, we refer to the fact that either $x s \wedge x t$ or $\overline{x s} \wedge \overline{x t}$ by " $x$ is not a splitter of $\{s, t\}$ ", denoted by $x \mid s t$. We need to prove that $x|s t \Leftrightarrow y| s t$. If none of $s$ and $t$ belong to $X$, that $X$ is a sesquimodule allows to conclude. If both $s$ and $t$ belong to $Y$, that $Y$ is a sesquimodule allows to conclude. By symmetry, the only remaining case is when $s \in X \cap Y$ and $t \notin X \cup Y$. In this case, let $u \in Y \backslash X$. Since $X$ is a sesquimodule, we already have $x|t u \Leftrightarrow y| t u$, but we would like the same property with vertex $u$ replaced by vertex $s$. For this, notice that $x \notin Y$, but $s, u \in Y$, and $Y$ is a sesquimodule. Therefore, $x \mid s u$. Likewise, $y \mid s u$. Then, combining the two latter facts and $x|t u \Leftrightarrow y| t u$ leads to the desired property.

Finally, a circular sesquimodule quotient node would be a complete one.

Remark 2. A 2-structure is roughly an edge-coloured complete digraph (see e.g. [10]). Graph modules can be generalised to clans of a 2-structure: a vertex subset $M$ of a 2 -structure is a clan if for all $x, y \in M$, for all $s \notin M$, the arcs 
$(s, x)$ and $(s, y)$ are of same colour, and the $\operatorname{arcs}(x, s)$ and $(y, s)$ are also of same colour (though the two colours may differ). Likewise, graph sesquimodules can also be generalised to sesquimodules of a 2 -structure as follows. $M$ is a sesquimodule of a 2 -structure if it holds two following conditions. For all $x, y \in M$, for all $s \notin M$, the arcs $(s, x)$ and $(s, y)$ are of the same colour. For all $x, y \in M$, for all $s, t \notin M,(x, s)$ and $(x, t)$ are of the same colour if and only if $(y, s)$ and $(y, t)$ are of the same colour. Then, one can check that the family of sesquimodules of any 2-structure is a union-difference family. Hence, for 2 -structures we have a similar decomposition theorem as what has been said for digraphs. However, the algorithm described in the next section wont apply.

Remark 3. We newly pointed out that the family of sesquimodules of a digraph is also closed under the intersection of its crossing members. Based on this, we improved the representation of the sesquimodules of a digraph $G=(V, A)$ from $O\left(|V|^{2}\right.$ ) (as in this paper) down to an $O(|V|)$ space decomposition tree. We also showed that the latter tree can be computed in polynomial time. All these new results can be found in [2]. This does not apply to the family of sesquimodules of a 2-structure since such a family could fail the closure under intersection of its crossing members.

\section{Polynomial Time Algorithm for Sesquimodular Decomposition}

This section describes a brute-force algorithm to compute in polynomial time the sesquimodular decomposition tree of a given digraph $G=(V, A)$. We divide the computation into two main steps, generalising the two-step scheme introduced by [3] for modular graph decomposition.

Definition 4 (Factoring Permutation). [3] A factoring permutation of a decomposition tree is the visit order of the leaves of the underlying decomposition tree by some depth-first graph search.

For sesquimodular decomposition tree, which is unrooted, we define the factoring permutation as a circular permutation. This notion dues its name to the fact that every node of the tree is a (circular) interval of the (circular) permutation. In the following, results of Section 4.1 can not be used unless we meet a certain notion of splitter. However, all the remaining is a scheme that can be used to compute the semi-strong tree of any arbitrary subset family. Indeed, the union-difference property will only be used for eventually typing the nodes.

\subsection{Computing a Factoring Permutation}

Like modular decomposition, we use a partition refinement technique (c.f. [16]) based on the notion of a splitter. There are two kinds of sesquimodule splitters:

- If there are $s \notin M$ and $x, y \in M$ with $(s, x) \in A$ (denoted by $s x$ ) and $(s, y) \notin A$ (denoted by $\overline{s y}$ ) then $M$ is not a sesquimodule. We say that vertex $s$ splits $x$ and $y$. 
- If there are $x, y \in M$ such that there are $s, t \notin M$ with $x \mid s t$ and $\overline{y \mid s t}$ then $M$ is not a sesquimodule, where $x \mid s t$ denotes $(x s \wedge x t) \vee(\overline{x s} \wedge \overline{x t})$ and $\overline{x \mid s t}$ denotes its negation. We say that vertex pair $(x, y)$ is a self-splitter.

Let us consider first category splitters. We begin with picking a vertex $x \in V$ and considering the ordered partition $\mathcal{P}=\left\{\overline{N^{+}(x)},\{x\}, N^{+}(x)\right\}$, which will be seen as an ordered circular partition. We then perform a round, which consists of performing the refinement operation (see right below) for all vertex $y \neq x$ until this modifies the partition $\mathcal{P}$. The round ends and we restart a new one whenever $\mathcal{P}$ is modified. If, through some whole round, the partition $\mathcal{P}$ remains unchanged for all $y \neq x$, then the process stops.

The refinement operation w.r.t. a vertex $y \neq x$ consists of splitting each part $P$ of the partition $\mathcal{P}$ into two parts: $P^{+}=P \cap N^{+}(y)$ and $\overline{P^{+}}=P \backslash N^{+}(y)$. The reorganisation of the split parts is as follows. Though obviously the part $Q=\{x\}$ of the partition $\mathcal{P}$ is not split, we have to consider whether $x \in N^{+}(y)$. Let $P$ and $R$ be the neighbour parts of $Q$ in $\mathcal{P}: \mathcal{P}=(\ldots, P, Q, R, \ldots)$. If $x \in N^{+}(y)$, replace $P$ with $\left(\overline{P^{+}}, P^{+}\right)$and replace $R$ with $\left(R^{+}, \overline{R^{+}}\right)$, else, replace $P$ with $\left(P^{+}, \overline{P^{+}}\right)$and $R$ with $\left(\overline{R^{+}}, R^{+}\right)$. If there are some empty set, we act as if they were present, but skip storing them to the partition list. Thus, the elements that $y$ "sees" the same way as how $y$ "sees" $x$ are locally stick together. We do the same processing for the parts before $P$ and those after $R$ in $\mathcal{P}$ (elements of same vision by $y$ are locally stick together). That there is in the initial partition an odd number of parts - actually 3 - guarantees no conflict when closing the circle.

At this point, we obtain a partition $\mathcal{P}$ such that for all vertex $v$, and for all part $P$ of the partition $\mathcal{P}, v$ is not a first category splitter of $P$. Since each refinement can be done in $O\left(\left|N^{+}(y)\right|\right)$ time (see [16]), a round takes $O\left(n^{2}\right)$ time, where $n=|V|$. Since each round decreases the partition $\mathcal{P}$ to a thinner partition, there are at most $n$ rounds. The total time is in $O\left(n^{3}\right)$.

We now consider second category splitters, with the computed partition $\mathcal{P}$. While there is in $\mathcal{P}$ some part $P$ containing a self-splitter $(x, y) \subseteq P$, replace $P$ with $\left(P_{x}, P_{y}\right)$, which is defined as follows. First, push $x$ in $P_{x}$ and $y$ in $P_{y}$. Let $s, t \notin P$ such that $x \mid s t$ and $\overline{y \mid s t}$. Then, for every other vertex $z$ of $P$, either $z \mid s t$ or $\overline{z \mid s t}$, and we push $z$ in $P_{x}$ or $P_{y}$ accordingly. Testing for self-splitters can be done by just testing all vertex quadruplets. This would globally cost $O\left(n^{5}\right)$ time.

At the end, we obtain a circularly ordered partition $\mathcal{P}=\left(P_{1}, \ldots, P_{k}\right)$ of unordered parts $P_{i}$ 's. Then, ordering arbitrary the $P_{i}$ 's results in a circular permutation of $V$, which is a factoring permutation.

\subsection{Computing the Decomposition Tree}

We here constantly need to test if a subset is member of the initial family. Let $\tau$ denotes the time for such a test. For digraphs, given a vertex subset, we can test in $\tau=O\left(n^{4}\right)$ time if the subset is member of the sesquimodule family by checking every vertex quadruplets. Then, the shape of the decomposition tree can easily be constructed in a brute-force manner as follows. Compute a factoring permutation. For each interval of the factoring permutation, test if it 
is a member of the initial family. For each pair of the latter members, if they cross, then remove both. Represent the remaining members in a cross-free treerepresentation as explained at the beginning of Section 2. Since there are at most $n^{2}$ intervals in a circular permutation, these operations take $O\left(n^{5}+n^{2} \tau\right)$ time.

The only remaining thing is to type the nodes. The main difficulty is how to test for nodes that are not simply-linked. Actually, we avoid this test by elimination of cases. For each internal node $i$ of the decomposition tree:

Compute the $2-$ graph of the quotient w.r.t. node $i$ (quadratic number of tests for membership). If this is a clique, a path, or a cycle, conclude accordingly, and stop. Compute all quasi-trivial members of the quotient. If there are more than one or none of such, report a prime node, and stop. Else either the node is prime or it is not simply-linked with that unique quasi-trivial member which is strong. Let $\{c\}$ be the complement of the unique quasi-trivial member. Assume node $i$ is not simply-linked and recursively compute the decomposition tree of the quotient excluding $\{c\}$ (refer to Section 2.2 for details). If the latter tree is anything except a single prime node then node $i$ effectively was not simplylinked, we conclude and stop. The latter tree is a single prime node. If there is some quasi-trivial member therein then node $i$ effectively was not simply-linked, we conclude and stop. Otherwise node $i$ was simply-linked. We report a prime node.

Without recursive calls the process is in $O\left(n^{3} \tau\right)=O\left(n^{7}\right)$ time. Then, an inductive argument similar to the proof of Theorem 2 gives an $O\left(n^{8}\right)$ time bound.

Theorem 4. The sesquimodular decomposition tree of a given digraph $G=$ $(V, A)$ can be computed in $O\left(|V|^{8}\right)$ time.

\section{Conclusion and Perspectives}

We have shown that union-difference families can be represented via a unique tree, and applied this result to a new directed graph decomposition. Of course the polynomial decomposition algorithm proposed here for this variation of modular decomposition has to be improved for a practical use. Another interesting investigation could be on the properties of the family of complements of members of a union-difference family. Such a family owns a quadratic representation straight from the result of union-difference families. However, their intrinsic properties are unclear, as the closure under difference does not behave symmetrically via complementary. Besides, representing families satisfying a number of closure operations remains an interesting question, and we are convinced that some other combinatorial decompositions can be expressed in this framework, as in [2].

Acknowledgements: The first author is grateful to S. Thomassé for helpful discussions and pointers.

\section{References}

1. http://hal-lirmm.ccsd.cnrs.fr/lirmm-00175766. 
2. B.-M. Bui-Xuan, M. Habib, and M. Rao. Representing partitive crossing Families and union-difference Families, with Application to Sesquimodular Decomposition. available at http://hal-lirmm.ccsd.cnrs.fr/lirmm-00199916.

3. C. Capelle and M. Habib. Graph decompositions and factorizing permutations. In 5th Israel Symposium on Theory of Computing and Systems (ISTCS'97), IEEE Computer Society, pages 132-143, 1997.

4. M. Chein, M. Habib, and M.C. Maurer. Partitive hypergraphs. Discrete Mathematics, 37(1):35-50, 1981.

5. D. A. Cohen, M. C. Cooper, and P. G. Jeavons. Generalising submodularity and Horn clauses: Tractable optimization problems defined by tournament pair multimorphisms. Technical Report CS-RR-06-06, Oxford University, 2006.

6. C. Crespelle. Représentations dynamiques de graphes. $\mathrm{PhD}$ thesis, Université Montpellier II, 2007.

7. W. Cunningham. Decomposition of directed graphs. SIAM Journal on Algebraic and Discrete Methods, 3:214-228, 1982.

8. W. Cunningham and J. Edmonds. A combinatorial decomposition theory. Canadian Journal of Mathematics, 32:734-765, 1980.

9. J. Edmonds and R. Giles. A min-max relation for submodular functions on graphs. Annals of Discrete Mathematics, 1:185-204, 1977.

10. A. Ehrenfeucht, T. Harju, and G. Rozenberg. The Theory of 2-Structures- A Framework for Decomposition and Transformation of Graphs. World Scientific, 1999.

11. M. G. Everett and S. P. Borgatti. Regular Equivalence: General Theory. Journal of Mathematical Sociology, 18:29-52, 1994.

12. J. Fiala and D. Paulusma. A complete complexity classification of the role assignment problem. Theoretical Computer Science, 349(1):67-81, 2005.

13. H. Gabow. Centroids, Representations, and Submodular Flows. Journal of Algorithms, 18(3):586-628, 1995.

14. J. Gagneur, R. Krause, T. Bouwmeester, and G. Casari. Modular decomposition of protein-protein interaction networks. Genome Biology, 5(8), 2004.

15. Tibor Gallai. Transitiv orientierbare Graphen. Acta Mathematica Academiae Scientiarum Hungaricae, 18:25-66, 1967.

16. M. Habib, C. Paul, and L. Viennot. Partition refinement techniques: An interesting algorithmic tool kit. International Journal of Foundations of Computer Science, 10(2):147-170, 1999.

17. W.-L. Hsu and R. M. McConnell. PC-trees and circular-ones arrangements. Theoretical Computer Science, 296:99-116, 2003.

18. J.-M. Lanlignel. Autour de la décomposition en coupes. $\mathrm{PhD}$ thesis, Université Montpellier II, 2001.

19. R.H. Möhring and F.J. Radermacher. Substitution decomposition for discrete structures and connections with combinatorial optimization. Annals of Discrete Mathematics, 19:257-356, 1984.

20. F. de Mongolfier and M. Rao. The bi-join decomposition. In 7th International Colloquium on Graph Theory (ICGT'05), 2005.

21. A. Schrijver. Combinatorial Optimization - Polyhedra and Efficiency. SpringerVerlag, 2003.

22. C. Semple and M. Steel. Phylogenetics. Oxford Lecture Series in Mathematics and Its Applications, number 24, 2003.

23. D. R. White and K. P. Reitz. Graph and Semigroup Homomorphisms on Networks of Relations. Social Networks, 5:193-234, 1983. 